\title{
EFECTO DEL ACEITE DE PALMA SOBRE EL CRECIMIENTO Y CAPACIDAD REPRODUCTIVA DE CARNEROS DE PELO PÚBERES
}

\author{
EFFECT OF PALM OIL ON GROWTH AND REPRODUCTIVE CAPACITY \\ IN PUBERTAL HAIR RAMS
}

\author{
Luna-Palomera, C. ${ }^{* *}$; Aguilar Cabrales, J.A. ${ }^{1}$ Peralta-Torres, J.A. ${ }^{1}$ \\ y Velázquez Martínez, J.R. ${ }^{1}$
}

\begin{abstract}
'Universidad Juárez Autónoma de Tabasco. División Académica de Ciencias Agropecuarias. Villahermosa, Tabasco. México. *dr_cluna@yahoo.com.mx
\end{abstract}

\section{PALABRAS CLAVE ADICIONALES}

Desarrollo corporal. Fertilidad. Reproducción. Trópico húmedo.

\section{RESUMEN}

Se evaluó la ganancia diaria de peso (GDP), calidad seminal, libido y capacidad de servicio en carneros de pelo, bajo pastoreo y suplementados con raciones conteniendo un $3 \%$ de aceite de palma (AP) o grasa animal (GA). Se utilizaron ocho corderos Black Belly $\times$ Kathadin y Pelibuey $\times$ Kathadin. A los animales se les suministró durante los primeros 60 días, $300 \mathrm{~g}$ por cabeza de una ración con $3 \%$ de GA, cantidad que se elevó a 500 $\mathrm{g}$ desde los 60 a los 120 días. En el grupo experimental GA se sustituyó por la misma proporción de aceite de palma. Cada 14 días se evaluó el peso vivo (PV), GDP, condición corporal (CC), circunferencia escrotal $(C E)$, perímetro torácico $(P T)$, altura a la cruz (AC) y largo de la cruz a la grupa (LCG). La calidad seminal se evaluó a partir de los 6 meses de edad con un peso promedio de 26,0 $\mathrm{kg}$. Durante siete semanas, se evaluó el volumen, concentración, motilidad masal e individual, \% de anormalidades primarias $\mathrm{y} \%$ de espermatozoides vivos. La libido y capacidad de monta se valoró con hembras estrogenizadas registrando el número de montas (NM), el número de servicios (NS), el número de montas por servicio (NMS), tiempo de reacción (TR) a las sucesivas montas $(1 \mathrm{M}, 2 \mathrm{M}$, $3 \mathrm{M})$ y servicios (1S, $2 \mathrm{~S}$ y $3 \mathrm{~S}$ ). Las variables se analizaron mediante un ANOVA. No se registraron diferencias entre AP y GA para las variables $P V$, GDP, PT, CC, AC y LCG. La calidad seminal, libido y capacidad de servicio fueron similares en ambos tratamientos. El número de servicios y el tiempo de

\section{AdDITIONAL KEYWORDS}

Body development. Fertility. Humid tropic. Reproduction.

reacción a la monta o al servicio fueron similares en ambos tratamientos sin embargo se observaron diferencias en el número de montas lo que se reflejó en el número de montas por servicio. El aceite de palma es una alternativa energética para la alimentación de carneros en el trópico que no afecta al crecimiento, calidad seminal y libido.

\section{SUMMARY}

The average daily gain (GDP), semen quality, libido, and serviceability in 8 hair rams (Black Belly $\times$ Kathadin and Pelibuey $\times$ Kathadin) supplemented with rations containing a $3 \%$ of palm oil (AP) or animal fat (GA) were studied during 120 days. During the first 60 days, lambs were fed with 300 $\mathrm{g} / \mathrm{animal} / \mathrm{d}$ of a diet with $3 \%$ of animal fat and, the following 60 days, each lamb received $500 \mathrm{~g}$ of feed. In the experimental group GA was replaced by AP. The average daily weight gain (GDP), body condition score (CC), scrotal circumference (CE), thoracic perimeter $(\mathrm{PT})$, height at withers $(\mathrm{AC})$ and length from the withers to the rump (LCG) were evaluated. The seminal quality was evaluated from 6 months of age and average weight of 26.0 $\mathrm{kg}$. During seven weeks, the volume, concentration, mass motility, individual primary abnormalities and living sperm percentage were assessed. To evaluate the libido and capacity of mating, the males were placed for 15 minutes with estrogenized females; number of mounts (NM), number of servi- 
ces (NS), number of mounts by service (NMS), reaction time (TR) to matings $(1 \mathrm{M}, 2 \mathrm{M}, 3 \mathrm{M})$ and services (1S, 2S, 3S), were recorded. The variables were analyzed by ANOVA. There were no differences among $A P$ and GA for the evaluated variables for PV, GDP, PT, CC, AC, and LCG. Semen quality, libido and serving capacity were similar. The number of services and reaction times for mount and service were also similar for both treatments, however diferents were observed in the number of mounts and so in number of mounts by service. The AP is an energetic alternative that can be considered in the feeding of rams in the tropics without affecting growth, the seminal quality and libido.

\section{INTRODUCCIÓN}

La evaluación de la capacidad reproductiva de un semental incluye su historia clínica, la calidad seminal, la libido y la capacidad de servicio. Entre 10 y $15 \%$ de los sementales sexualmente activos presentan alguna disminución en su capacidad reproductiva o fertilidad cuestionable (Seaman, 2004; Schoenian, 2006). Estas cifras pueden alcanzar hasta un $25 \%$ (Castrillejo, 1990), por lo que la productividad y rentabilidad de empresa ovina puede verse afectada negativamente si son utilizados como reproductores.

Las pruebas de libido y capacidad de servicio en las evaluaciones de sementales a nivel de campo generalmente no se incluyen, por lo que un semental puede estar clínicamente sano y con muy buena calidad seminal, pero si la libido y capacidad de servicio es baja, la eficiencia reproductiva del rebaño será baja (Perkins et al., 1992; Ibarra et al., 1999). Por lo anterior, es importante documentar el desempeño productivo, la calidad seminal, y la libido y capacidad de servicio de los carneros candidatos a sementales desde edades tempranas a fin de emitir recomendaciones para el manejo reproductivo.

En latitudes al norte $\left(19^{\circ}\right)$ se reporta actividad reproductiva estacional ligada al fotoperiodo en las ovejas y por lo tanto en el carnero lo que se refleja en los perfiles hormonales a través del año (Schanbachern y Lunstra, 1976), con desempeño reproductivo más activo en días cortos y menos activo en días largos (Lincoln y Davidson, 1977). En las ovejas Pelibuey y Black Belly y sus cruzas en el trópico húmedo se reportan grupos que no presentan estacionalidad reproductiva como las razas de zonas templadas (Hinojosa et al., 2005; Hinojosa y Oliva, 2009), lo cual es posible se aplique a los sementales ya que estas razas se reportan como no estacionales (Rosa y Bryant, 2003), por lo que su fertilidad debe evaluarse en épocas de días largos debido a que la pérdida de actividad reproductiva es también marcada en los carneros (OlivaHernández et al., 2002).

Por otra parte, en comunidades rurales marginadas, la suplementación del carnero en preparación para las épocas de monta es casi nula debido a los altos costos de granos y concentrados. El cultivo de palma de aceite (Elaeis guineensis) en el trópico mexicano permite producir entre 4 y 7 toneladas por hectárea de aceite crudo (Ortega y Ochoa, 2003) el cual puede obtenerse de forma artesanal (Velázquez y Gómez, 2010). En comunidades rurales el aceite crudo de palma africana es una fuente natural energética (9 calorías por gramo) disponible y de bajo costo comparada con los aceites de maíz, soya, cártamo o grasas de origen animal. Destaca su composición balanceada de ácidos grasos, con $50 \%$ de ácido oleico, principal componente del aceite de oliva que en combinación con el palmítico permite reducir significativamente los niveles de colesterol LDL (Jewett, 2002) y su eficiente transformación esteroidogénica (King y LaVoie, 2009). Su contenido de antioxidantes tales como tocoferoles y tocotrienoles (es el único que contiene 8 isómeros de la vitamina E) en concentraciones entre 700 y 1000 ppm, y carotenoides (pro vitamina A y D) con concentraciones entre 500 y 700 ppm (Pantzaris, 2000; Velázquez y Gómez, 2010) 


\section{ACEITE DE PALMA EN CARNEROS DE PELO PÚBERES}

le permite tener propiedades funcionales antioxidantes importantes (Khor y Raajeswari, 2001) sobre todo en beneficio del desempeño productivo y reproductivo (Strzezek et al., 2004) pero que no se conoce su efecto sobre la calidad seminal (volumen, concentración y viabilidad espermática) y desempeño reproductivo (libido y capacidad de servicio).

El objetivo del estudio fue evaluar el crecimiento, la calidad seminal, la libido y capacidad de servicio en corderos de pelo suplementados con aceite de palma en sustitución de grasa animal, en épocas de días largos en el trópico húmedo.

\section{MATERIAL Y MÉTODOS}

\section{UBICACIÓNGEOGRÁFICA}

El experimento se llevó a cabo en la posta de producción animal de la División Académica de Ciencias Agropecuarias, Universidad Juárez Autónoma de Tabasco localizada a $17^{\circ} 46^{\prime} \mathrm{Ny} 92^{\circ} 57^{\prime} \mathrm{O}$ (Cámara etal., 2008). El estudio se realizó entre los meses de marzo y agosto de 2009. En promedio, la temperatura ambiente es de $28,6{ }^{\circ} \mathrm{C}$ y la precipitación pluvial en año seco de 1309 $\mathrm{mm}$.

\section{Animales y mANEJO}

Se utilizaron ocho corderos comerciales procedentes de partos simples cruzados de Kathadin $\times$ Black Belly $(\mathrm{KT} \times \mathrm{BB} ; \mathrm{n}=4) \mathrm{y}$ Katahdin $\times$ Pelibuey $(\mathrm{KT} \times \mathrm{PB} ; \mathrm{n}=4)$, con un peso inicial de $16 \pm 1,9 \mathrm{~kg}, 2,5$ meses de edad, los cuales se asignaron a los tratamientos tomando como bloque su origen racial, sin que éste fuera un factor relevante en la evaluación de las variables de respuesta.

Los corderos se alimentaron con base en pastoreo de Cynodon plectostachyus y una complementación alimenticia (tratamientos), que contenía aceite crudo de palma (AP) o grasa animal de pollo (GA) administrada después del pastoreo.

En promedio cada cordero recibió aproximadamente $1,5 \%$ de su peso vivo: $300 \mathrm{~g}$ animal $^{-1} \mathrm{~d}^{-1}$ del complemento (3 Mcal/kg y $20 \%$ PB) conteniendo $3 \%$ de aceite de palma africana (Elaeis guineensis) o de grasa de pollo por un periodo inicial de 60 días y 500 $\mathrm{g}$ animal ${ }^{-1} \mathrm{~d}^{-1}$ durante un segundo periodo de igual duración.

Los procedimientos experimentales con los animales fueron aprobados por el Comité de Ciencia Animal de la Universidad Juárez Autónoma de Tabasco, en acuerdo con las leyes de bienestar animal en México.

\section{DESARROLLODECORDEROS}

Cada 14 días se registraron las siguientes variables: peso vivo (PV), ganancia diaria de peso (GDP), condición corporal (CC: por palpación de la cantidad de grasa en las apófisis espinosas, apófisis transversas y músculos del lomo asignando valores de 1 , emaciados, a 5, obesos); circunferencia escrotal (CE: perímetro en la región ecuatorial a la altura de la rete testis usando un testímetro); perímetro torácico (PT: por la región interescapular-región esternal); altura a la cruz (AC: desde el suelo hasta el punto más alto de la cruz) y largo de la cruz a la grupa (LCG: desde el punto más alto de la cruz hasta la tuberosidad coxal).

\section{Calidad SEMINAL}

Después de 100 d de suplementación (entre 6 y 7 meses de edad y 26,0 kg de PV promedio), se expuso a los corderos a presenciar las montas realizadas por un macho entrenado a hembras sujetas a un potro de monta. Posteriormente, se dejó a los corderos montar en forma individual. Antes de la monta se les realizó un lavado prepucial con una solución desinfectante, se permitieron tres intentos de monta antes de la colecta para lograr mayor interés de los sementales y una mejor calidad del semen. El semen se obtuvo con vagina artificial a $40-41{ }^{\circ} \mathrm{C}$. Se lavó la camisa con jabón neutro, se enjugó y secó con aire caliente. Se tomaron dos muestras por día a una frecuencia de dos veces por semana hasta completar 28 eyaculados por carnero. 
El semen fue examinado inmediatamente, evaluando volumen y color en tubos graduados de $1,5 \mathrm{ml}$.

La motilidad masal se determinó inmediatamente después de la obtención sobre una muestra de $5 \mu \mathrm{L}$ de semen en un portaobjetos atemperado en platina caliente; se observó en contraste de fases con objetivo 10X, asignando valores de 0 (motilidad nula) a 5 (motilidad de remolinos vigorosa de movimientos en masa).

La motilidad individual se determinó sobre $5 \mu \mathrm{L}$ de semen con cantidad similar de solución salina en portaobjetos atemperado. En microscopio de contraste de fases (objetivo 40X) se asignaron valores de 0 a $100 \%$ en función de la motilidad individual progresiva y el vigor con el cual atravesaban el campo los espermatozoides.

El porcentaje de espermatozoides vivos se realizó sobre frotis teñido con eosinanigrosina secada a temperatura ambiente y contando 100 espermatozoides en el campo, considerando muertos los que se tiñeron total o parcialmente.

Sobre el frotis se observaron, las anormalidades de estructura y morfología de 100 células, y se determinó el número de espermatozoides con cabezas sueltas, colas enrolladas y colas dobles.

La concentración se determinó por el método de hemocitómetro haciendo diluciones 1:200 (Salamon et al., 1990; De Lucas y Arbiza, 2006).

Para el conteo espermático se tomó una muestra de semen con un hemocitómetro hasta la marca de 5 y posteriormente se llenó la pipeta hasta la marca de 101 con solución salina y rosa de bengala. Se agitó manualmente 30 a 40 veces, eliminando las tres primeras gotas, la siguiente se colocó en la cámara de Neubauer y se procedió al conteo en ambos lados de la cámara.

\section{LIBIDO Y CAPACIDAD DESERVICIO}

La evaluación se realizó al final del periodo de obtención de semen con la metodología de Kilgour (1985) modificada con la ayuda de hembras en celo inducido con aplicaciones de $0,06 \mathrm{mg}$ de cipionato de estradiol (ECP, Pharmacia \& Upjhon $\left.{ }^{\circledR}\right)$ $\mathrm{kg}^{-1}$ de peso corporal. Se realizaron dos pruebas a intervalo de una semana, empleando una oveja por semental y, durante 15 minutos, se evaluaron las siguientes variables: Número de servicios (NS), número de veces que el macho realiza la intromisión con eyaculación determinada a través del golpe de riñón; Número de montas (NM), número de veces que el macho eleva los miembros anteriores apoyando el pecho sobre la grupa pudiendo haber introducción del pene sin eyaculación; Tiempo de reacción al servicio (TR1S, TR2S, TR3S) o a la monta(TR1M, TR2M, TR3M) es el intervalo entre el inicio de la prueba y el acto y Número de montas por servicio (NMS).

\section{ANÁLISIS ESTADÍSTICO}

Se realizó un análisis de varianza bajo un modelo mixto por el procedimiento MIXED de SAS (1999) para las variables PV final, GDP a los 120 días de pruebas, CC, CE, PT, AC y LCG se consideraron efectos fijos tratamiento, período de medición e interacciones de primer orden y como efectos aleatorios el bloque $(\mathrm{KT} \times \mathrm{BB}$ o KT $\times \mathrm{PB})$ y semental dentro de tratamiento. Para las variables volumen, motilidad masal (MM), motilidad individual (MI), espermatozoides vivos (\%), espermatozoides con anormalidades primarias (\%) y concentración, se consideraron como efectos fijos el tratamiento, número de eyaculado (4 por semental semana ${ }^{-1}$ ), semana de muestreo (7 semanas) e interacciones de primer orden. Los efectos aleatorios fueron bloque y semental dentro de tratamiento. Adicionalmente se realizaron correlaciones de Spearman entre las variables zoométricas mediante PROC CORR de SAS (1999).

Para las variables relacionadas con la libido se tuvieron en cuenta como efectos fijos el de tratamiento y el número de prueba por semental y como aleatorios, el bloque y semental dentro de tratamiento. 


\section{ACEITE DE PALMA EN CARNEROS DE PELO PÚBERES}

Tabla I. Valores medios de características zoométricas entre carneros de pelo púberes desarrollados con un suplemento con aceite de palma (AP) o grasa animal (GA). (Mean value of zoometric characteristic between young hair rams growing with a supplement with palm oil (AP) or animal fat $(G A))$.

\begin{tabular}{|c|c|c|c|c|c|c|c|c|}
\hline & PVI & PVF (kg) & $\operatorname{GDP}\left(\mathrm{g} \mathrm{d}^{-1}\right)$ & CC (1 a 5) & $\mathrm{CE}(\mathrm{cm})$ & $A C(\mathrm{~cm})$ & $\mathrm{PT}(\mathrm{cm})$ & LCG $(\mathrm{cm})$ \\
\hline AP & $15,21 \pm 0,0^{a}$ & $26,2 \pm 1,9^{a}$ & $101,4 \pm 17,9^{\mathrm{a}}$ & $3,1 \pm 0,1^{\mathrm{a}}$ & $28,4 \pm 1,9^{a}$ & $58,4 \pm 1,5^{\mathrm{a}}$ & $67,2 \pm 1,6^{\mathrm{a}}$ & $57,5 \pm 1,2^{a}$ \\
\hline GA & $15,71 \pm 0,9^{a}$ & $26,8 \pm 1,9^{a}$ & $112,2 \pm 17,9^{a}$ & $3,1 \pm 0,1^{\mathrm{a}}$ & $28,4 \pm 1,9^{a}$ & $58,4 \pm 1,5^{\mathrm{a}}$ & $67,9 \pm 1,6^{a}$ & $57,8 \pm 1,2^{a}$ \\
\hline
\end{tabular}

abLiterales diferentes dentro de columna señalan diferencias estadísticas $(p<0,05)$.

$\mathrm{PVI}=\mathrm{Peso}$ vivo inicial; $\mathrm{PVF}=\mathrm{Peso}$ vivo final; $\mathrm{GDP}=$ Ganancia diaria de peso; $\mathrm{CC}=$ Condición corporal; $\mathrm{CE}=$ Circunferencia escrotal; $\mathrm{AC}=$ altura a la cruz; $\mathrm{PT}=$ perímetro torácico; $\mathrm{LCG}=$ largo de la cruz a la grupa.

\section{RESULTADOSYDISCUSIÓN}

\section{DESARROLLOCORPORALDELOS CORDEROS}

Los tratamientos no influyeron sobre los pesos vivos inicial y final por tanto no hubo diferencias en la GDP al final de los 120 días (tabla I) siendo sus cifras similares a las reportadas por Frías et al. (2011) con ganancias diarias de peso de $101 \mathrm{~g} \mathrm{~d}^{-1}$ para cordero Pelibuey, pero superiores a las reportadas por Díaz-Quevedo (2010) en corderos híbridos $\mathrm{PB}$ o $\mathrm{BB} \times$ Dorper en las cuales reportó ganancias máximas de 73,7 $\mathrm{g} \mathrm{d}^{-1}$.

Los coeficientes de correlación entre las variables zoométricas fueron positivos y altos $(\mathrm{p}<0,01)$ para las variables peso y CC $(0,68)$, pesos y CE $(0,79)$, peso y AC $(0,74)$, peso y LCG $(0,87)$, las cuales están fuertemente asociadas con el crecimiento y desarrollo corporal de los corderos.

\section{CALIDAD SEMINAL}

Los resultados (tabla II) no muestran diferencias estadísticas para CE, volumen, MM, concentración y anormalidades. No obstante se observó menor MM y MI $(\mathrm{p}<0,05)$ en las primeras semanas de evaluación (3,0 y $62 \%$ para AP, y 3,2 y $60 \%$ para $\mathrm{GA}$, respectivamente) que puede atribuirse a la edad de los corderos ( 6 a 8 meses) y el establecimiento de su madurez sexual al momento de las evaluaciones. En términos generales, los valores encontrados para las variables relacionadas con la calidad seminal están dentro de los rangos reportados por otros autores (Hulet y Shelton, 1980), y son comparables con los encontrados en Yucatán (Victoria et al., 2009) en machos Pelibuey y Kathadin entre 6 y 12 meses de edad. Por otra parte, los resultados encontrados para concentración espermática en

Tabla II. Calidad seminal en carneros púberes de pelo suplementados con aceite de palma o grasa animal. (Seminal quality in young hair rams supplemented with palm oil or animal fat).

\begin{tabular}{cccccccc}
\hline CE & $\begin{array}{c}\text { VOL } \\
(\mathrm{mL})\end{array}$ & $\begin{array}{c}\text { MM } \\
(0 \mathrm{a} 5)\end{array}$ & $\begin{array}{c}\text { Ml } \\
(\%)\end{array}$ & $\begin{array}{c}\text { Vivos } \\
(\%)\end{array}$ & $\begin{array}{c}\text { CONCEN } \\
\times 10^{6} \mathrm{~mL}^{-1}\end{array}$ & $\begin{array}{c}\text { ANORM } \\
\%\end{array}$ \\
\hline AP & $30,5 \pm 1,2^{\mathrm{a}}$ & $0,85 \pm 0,1^{\mathrm{a}}$ & $3,7 \pm 0,1^{\mathrm{a}}$ & $75,3 \pm 1,3^{\mathrm{a}}$ & $89,7 \pm 3,0^{\mathrm{a}}$ & $3,592 \pm 199,2^{\mathrm{a}}$ & $2,7 \pm 0,7^{\mathrm{a}}$ \\
$\mathrm{GA}$ & $29,7 \pm 1,2^{\mathrm{a}}$ & $0,91 \pm 0,1^{\mathrm{a}}$ & $3,8 \pm 0,1^{\mathrm{a}}$ & $74,5 \pm 1,3^{\mathrm{b}}$ & $92,1 \pm 3,0^{\mathrm{a}}$ & $3,361 \pm 199,2^{\mathrm{a}}$ & $2,5 \pm 0,7^{\mathrm{a}}$ \\
\hline
\end{tabular}

ab Literales diferentes dentro de columna son estadísticamente diferentes $(p<0,05)$.

$\mathrm{CE}=$ Circunferencia escrotal; $\mathrm{VOL}=$ Volumen; $\mathrm{MM}=$ Motilidad masal; $\mathrm{MI}=$ Motilidad individual; $\mathrm{CONCEN}=$ Concentración; ANORM= anormalidades primarias. 
LUNA-PALOMERA, AGUILAR CABRALES, PERALTA-TORRES Y VELÁZQUEZMARTÍNEZ

Tabla III. Libido en carneros púberes complementados con aceite de palma o grasa animal. (Libido in young rams supplemented with palm oil or animal fat).

\begin{tabular}{lccccr}
\hline & Número montas & TR1M $(\min )$ & TR2M $(\min )$ & TR3M $(\min )$ & TR4M (min) \\
\hline AP & $8,37 \pm 0,9^{\mathrm{a}}$ & $1,08 \pm 0,6^{\mathrm{a}}$ & $1,6 \pm 1,0^{\mathrm{a}}$ & $3,76 \pm 1,5^{\mathrm{a}}$ & $5,53 \pm 1,8^{\mathrm{a}}$ \\
GA & $4,88 \pm 0,9^{\mathrm{b}}$ & $0,23 \pm 0,6^{\mathrm{a}}$ & $2,0 \pm 1,0^{\mathrm{a}}$ & $4,36 \pm 1,6^{\mathrm{a}}$ & $6,76 \pm 2,2^{\mathrm{a}}$ \\
\hline
\end{tabular}

ab Literales diferentes dentro de columna son estadísticamente diferentes $(p<0,05)$.

TR1M, TR2M y TR3M= tiempo de reacción a la primera, segunda y tercera monta.

esta época del año están por debajo a los reportados por Aguirre et al. (2007) en carneros Pelibuey adultos con valores de 4020 y $4400 \times 10^{6}$ espermatozoides $\mathrm{mL}^{-1}$ en días largos y cortos respectivamente bajo un esquema de dos eyaculados por semana, comparados a los encontrados.

A fin de aprovechar la disponibilidad del aceite de palma y su contenido de ácidos grasos saturados e insaturados, es importante considerar en experiencias futuras investigar con diferentes niveles de inclusión de aceite y protegerlo de la hidrólisis a su paso por el rumen a fin de evitar su potencial peroxidación, y formación de radicales libres reactivos e inestables a nivel de membrana celular, asociados con la oxidación y muerte celular, lo cual explicaría en parte el menor porcentaje de espermatozoides vivos en este trabajo, y por lo tanto la ausencia de diferencias estadísticas en las demás variables relacionadas. En este sentido De Graaf et al. (2007) reportan menor porcentaje de espermatozoides viables y con acrosoma intacto en carneros alimen- tados con dietas que incluyeron aceite de oliva (alto en ácido graso oleico) y aceite de girasol (alto en ácido graso linoleico). Mientras que Strzezek et al. (2004) en cerdos alimentados con dietas que incluyeron ácidos grasos insaturados, observaron una mejora de las características seminales, tales como el porcentaje de espermatozoides con membrana plasmática intacta y la resistencia osmótica de la membrana acrosomal, efecto que se considera pueda ser similar en rumiantes si se protegen los ácidos grasos del aceite de palma a su paso por el rumen.

\section{LIBIDOY CAPACIDAD DE SERVICIO}

No se encontraron diferencias estadísticas entre tratamientos $(p>0,05)$ con respecto a las variables evaluadas (tablas III y IV) salvo número de montas y montas por servicio. A pesar de que se trata de sementales púberes y sin experiencia sexual, la libido mostrada por los animales (tabla III) en ambos tratamientos es satisfactoria ya que registraron de 5 a 8 montas en 15 minutos.

La capacidad de servicio evaluada a tra-

Tabla IV. Capacidad de servicio en carneros púberes complementados con aceite de palma o grasa animal. (Service ability in young rams supplemented with palm oil or animal fat).

\begin{tabular}{lccccc}
\hline & NS & Montas/Servicio & TR1S $(\min )$ & TR2S $(\min )$ & TR3S (min) \\
\hline AP & $3,0 \pm 0,3^{\mathrm{a}}$ & $3,04 \pm 0,3^{\mathrm{a}}$ & $3,04 \pm 1,9^{\mathrm{a}}$ & $5,63 \pm 1,9^{\mathrm{a}}$ & $6,56 \pm 2,6^{\mathrm{a}}$ \\
GA & $2,6 \pm 0,3^{\mathrm{a}}$ & $1,94 \pm 0,3^{\mathrm{b}}$ & $1,27 \pm 1,9^{\mathrm{a}}$ & $5,50 \pm 1,9^{\mathrm{a}}$ & $8,50 \pm 2,7^{\mathrm{a}}$
\end{tabular}

abLiterales diferentes dentro de la misma columna son estadísticamente diferentes $(p<0,05)$.

NS= Número de servicios; TR1S, TR2S y TR3S= tiempo de reacción al primero, segundo y tercer servicio.

Archivos de zootecnia vol. 62, núm. 237, p. 50. 


\section{ACEITE DE PALMA EN CARNEROS DE PELO PÚBERES}

vés del número de servicios fue satisfactoria en ambos tratamientos (tabla IV), aunque con diferencias $(\mathrm{p}<0,05)$ entre AP y GA para $\mathrm{NM}(8,37$ vs. 4,88), NMS $(3,04$ vs. 1,9$)$ respectivamente, asociado con una mayor libido debida posiblemente a mayores niveles de testosterona (Luna et al., 2011) en los carneros suplementados con AP. El desempeño para esta característica es superior a lo reportado por Ibarra et al. (1999) en carneros adultos con experiencia sexual previa de las razas Corriedale, Merino y Milchschaf en un tiempo de 20 minutos.

La circunferencia escrotal, calidad del semen y libido (altamente heredable) se considera predictiva de la capacidad de servicio (Perry y Patterson, 2009). Perkins et al. (1992) reportan que carneros con alta capacidad de servicio montaron más ovejas en un periodo de 9 días $(97,4 \%)$ que carneros de baja capacidad $(32,2 \%)$, resultando un mayor número hembras paridas $(89,5 \%$ vs. 36,0 \%), y de corderos nacidos (1,85 vs. $0,78)$. Los machos con alta capacidad de

\section{BIBLIOGRAFÍA}

Aguirre, V.; Orihuela, A. and Vázquez, R. 2007. Effect of semen collection frequency on seasonal variation in sexual behaviour, testosterone, testicular size and semen characteristics of tropical hair rams (Ovis aries). Trop Anim Health Pro, 39: 271-277.

Cámara-Córdova, J.; Nava A., J.; Flores B., R. Manjarres M., B. y Mendoza P., D. 2008. Caracterización ambiental del Rancho-Escuela de la DACA-UJAT. En: Cámara-Córdova, J.; R. Flores B. (Eds). Ciencia Animal Mesoamericana $1^{\underline{a}}$ ed. Col. José N. Rovirosa. Universidad Juárez Autónoma de Tabasco. Villahermosa, Tabasco.

Castrillejo, A. 1990. Relevamiento clínico de la aptitud reproductiva en carneros. Veterinaria, 26: 15-32.

De Graaf, S.; Peake, K.; Maxwell, W.M.C.; O'Brien, J. and Evans, G. 2007. Influence of supplementing diet with oleic and linoleic acid on the freezing ability and sex-sorting parameters of ram semen. Livest Sci, 110: 166-173.

De Lucas Tron, J. y Arbiza Aguirre, S.I. 2006. servicio dieron más servicios en un tiempo determinado, lo cual coindice con los resultados obtenidos en la presente investigación, ya que los carneros dieron al menos cuatro servicios en 15 minutos. Por lo anterior, la libido y capacidad de servicio deben ser incluidas como rasgos a ser evaluados en los carneros candidatos a sementales.

El desarrollo corporal, la calidad seminal, libido y capacidad de servicio en sementales jóvenes complementados con AP o GA fue similar. El desempeño reproductivo de los sementales fue satisfactorio en ambos tratamientos para la época del año evaluada. El AP puede ser una alternativa para la complementación energética en carneros candidatos a sementales para pequeños criadores que disponen de este recurso en el trópico. Es importante en futuras experiencias considerar proteger las grasas del aceite de palma de la hidrólisis a su paso por el rumen, a fin de determinar si su sobrepaso impacta significativamente el desempeño reproductivo de los carneros.

Sistema de apareamiento e inseminación artificial en ovinos. Manual Técnico. UNAM. México, D.F.

Díaz Quevedo, V. 2010. Efecto del vitafer en el comportamiento de ovinos en finalización en pastoreo suplementados con sachapulido. Tesis de maestría. Colegio de postgraduados. $\mathrm{H}$. Cárdenas. Tabasco. México. 51 pp.

Frías, J.C.; Aranda, E.M.; Ramos, J.A.; Vázquez, C. y Díaz, P. 2011. Calidad y rendimiento en canal de corderos en pastoreo suplementados con caña de azúcar fermentada. Av Invest Agropec, 15: 33-44.

Hinojosa-Cuéllar, J.A.; García-Méndez, G. y OlivaHernández, J. 2005. Comportamiento reproductivo de ovejas Blackbelly y sus cruzas con Pelibuey, Dorper y Katahdin en Centla, Tabasco, México. IV Seminario de producción de ovinos en el trópico. Universidad Juárez Autónoma de Tabasco. Villahermosa. 2 y 3 de diciembre. Tabasco, México. pp. 8-13.

Hinojosa-Cuéllar, J.A. y Oliva-Hernández, J. 2009.

Archivos de zootecnia vol. 62, núm. 237, p. 51. 
Distribución de partos por estación en ovejas de razas de pelo y cruces en un ambiente tropical húmedo. Rev Cient, FCV-LUZ. XIX: 288-294.

Hulet, C.V. and Shelton, M. 1980. Sheep and goat. In: Hafez ESE editors. Reproduction in farm animals. $4^{\text {th }}$ edition. Lea and Febiger. Philadelphia. USA.

Ibarra, D.; Laborde, D.; Olivera, J.; Van Lier, E. y Burgueños, J. 1999. Comparación de tres pruebas para medir la capacidad de servicio en carneros adultos. Arch Med Vet, 31: 189-196.

Jewett, B. 2002. MUFA versus PUFA. Scientists disagree on nutricional importance of these fatty acids. INFORM, 13: 376-379.

Khor, H.T. and Raajeswari, R. 2001. Red palm oil, vitamin $A$, and the antioxidant enzymes. In: micronutrientes and health molecular biological mechanisms. Ed. Nesaretnam, K. and Packer, L. AOCS Press. Champaign, Illinois. USA. pp. 299-312.

Kilgour, J. 1985. Mating behavior of rams in pens. Aust J Exp Agr, 25: 298-305.

King, S.R. and LaVoie, H.A. 2009. Regulation of early steps in gonadal steroidogenesis. In: Reproductive endocrinology: A molecular approach. P.J. Chedrese (Ed.). Springer. N.Y. USA. $361 \mathrm{pp}$.

Lincoln, G. and Davidson, W. 1977. The relationship between sexual and aggressive behavior, and pituitary and testicular activity during the seasonal sexual cycle rams, and the influence of photoperiod. J Reprod Fertil, 49: 267-276.

Luna Palomera, C.; Velázquez Martínez, J.R.; De la Cruz Hernández, J.C.; Blé Castillo, J.L.; Peralta Torres, J.A.; Berumen Alatorre, A.C. y Aguilar Cabrales, J.A. 2011. Calidad seminal y metabolitos lipídicos en carneros de pelo suplementados con aceite de palma (Elaeis guineensis). XXIII Reunión Científica-Tecnológica Forestal y Agropecuaria Tabasco 2011. 24 y 25 de Noviembre. Villahermosa, Tabasco. México. pp. 118-122.

Oliva-Hernández, J.; Mora-Morelos, H.; Sánchez, M.J.M. y Hinojosa-Cuéllar, J.A. 2002. Producción de ovinos de pelo en Tabasco. Condiciones climáticas y apareamiento. Kuxulkab'. 8: 8-23.

Ortega, C. y Ochoa, R. 2003. La palma africana, una oleaginosa de ambiente tropical. Rev Claridades Agropecuarias, 122: 3-17.

Pantzaris, T.P. 2000. Pocketbook of palm oil uses. $5^{\text {th }}$ Ed. Malaysian palm oil board (MPOB). Kuala Lumpur. Malaysia.

Perry, G. y Patterson, D. 2009. Determinación de la fertilidad reproductiva de toros padres Hereford, Bs. As., 71: 52-59. http://produccion bovina.com/informacion_tecnica/cria_toros/22determinacion_fertilidad.pdf (16/06/2010).

Perkins, A.; Fotzgelard, J.A. and Price, E.O. 1992. Sexual performance of rams in serving capacity test predicts success in pen breeding. $J$ Anim Sci, 70: 2722-2725.

Rosa, H.J.D. and Bryant, M.J. 2003. Seasonality of reproduction in sheep. Small Ruminant Res, 48: 155-171.

SAS. 1999. User's Guide. Statistical Analysis System Institute. Cary, N.C. USA.

Salamon, S.; Evans, G. y Maxwell, W.M.C. 1990. Inseminación artificial de ovejas y cabras. Editorial Acribia, S.A. Zaragoza. España.

Schambacher, B. and Lunstra, D. 1976. Seasonal changes in sexual activity an serum levels of $\mathrm{LH}$ and testosterone in Finnish Landrace and Suffolk rams. J Anim Sci, 43: 644-650.

Schoenian, S. 2006. Reproduction in the ram. Sheep 201: A beginner's guide to raising sheep. Western Maryland Research and Education Center Maryland Cooperative Extension. http:/ /www.sheep101.info/201/ramrepro.html (20/ 06/2010).

Seaman, J.T. 2004. Fertility testing of rams. Flock health. NSW Department of Primary Industries. http://www.dpi.nsw.gov.au/_data/assets/ pdf_file/0010/179785/fertility-test-rams.pdf (20/ 06/2010).

Strzezek, J.; Fraser, L.; Kukliñska, M.; Dziekoñska, A. and Lecewicz, M. 2004. Effects of dietary supplementation with polyunsaturated fatty acids and antioxidants on biochemical characteristics of boar semen. Reprod Biol, 4: 271-87.

Velázquez-Martínez, J.R. y Gómez-Vázquez, A. 2010. Palma africana em Tabasco. Ed. José N. Rovirosa. Villahermosa, Tabasco, México. 225 $\mathrm{pp}$.

Victoria, S.J.; Zapata, K.I.; Navarrete, L.; Erosa, D.S.; Ávilez, A.V.; Pérez, L.P. y Aguilar, L.A. 2009. Producción espermática en ovinos entre los seis y doce meses de edad. III Congreso Internacional de las Ciencias Agropecuarias. La Habana. Cuba. 